\title{
PERIODIC SOLUTIONS OF CERTAIN ABSTRACT WAVE EQUATIONS
}

\author{
MICHAL FEČKAN \\ (Communicated by Barbara Lee Keyfitz)
}

\begin{abstract}
Existence results of periodic solutions of certain abstract, weakly nonlinear, wave equations are given when eigenvalues of linear parts of those equations are incommensurable to the time period.
\end{abstract}

\section{INTRODUCTION}

Recently, J. Mawhin and K. Ben-Naoum [1] and P. J. McKenna [2] investigated the existence of periodic solutions of one-dimensional wave equations when the ratio between the space length and the period was irrational. Generally, problems of those kinds lead to problems of small divisors, and for this reason, it is very hard to study such problems. On the other hand, we can very easily study these problems for specific irrational numbers of the ratio. These irrational numbers can be nicely characterized in notions of the number theory $[1,2]$.

The purpose of this paper is to proceed in the direction of these papers for more general wave equations. We shall study the equation

$$
u_{t t}+A u=\varepsilon f(u, t) \text {, }
$$

where $A$ is a selfadjoint, unbounded linear operator with eigenvalues $\lambda_{1}<$ $\lambda_{2}<\cdots \rightarrow \infty, \varepsilon$ is a small parameter, and $f$ is $T$-periodic in $t \in \mathbb{R}$. By a $T$-periodic solution of (1.1) we mean a weak solution as specified below.

In the first part of this paper, we assume $\lambda_{i} \neq 0 \quad \forall i=1,2, \ldots$. We shall prove the following result under additional assumptions on $A, f$.

Theorem 1.1. Assume there exists a constant $c>0$ such that

$$
\left|\alpha^{2}-\frac{m^{2}}{\lambda_{i}}\right| \geq \frac{c}{\lambda_{i}} \quad \forall m \in \mathscr{N}, \forall \lambda_{i}>0,
$$

where $\alpha=T / 2 \pi$. Then (1.1) has a weak $T$-periodic solution for any $\varepsilon$ small.

Note that condition (1.2) is satisfied provided

$$
\left|\alpha-\frac{m}{\sqrt{\lambda_{i}}}\right| \geq \frac{c}{\lambda_{i}} \quad \forall m \in \mathscr{N}, \forall \lambda_{i}>0,
$$

Received by the editors May 12, 1993.

1991 Mathematics Subject Classification. Primary 35B10, 35B20, 11 A55. sions.

Key words and phrases. Periodic solutions, abstract wave equations, continued fraction expan- 
for a constant $c>0$. Indeed, we have by $(1.3)$

$$
\left|\alpha^{2}-\frac{m^{2}}{\lambda_{i}}\right|=\left|\alpha-\frac{m}{\sqrt{\lambda_{i}}}\right| \cdot\left|\alpha+\frac{m}{\sqrt{\lambda_{i}}}\right| \geq \alpha \frac{c}{\lambda_{i}} .
$$

For the one-dimensional operator

$$
A u=-u_{x x}, \quad u(0)=u(\pi)=0, \quad u \in C^{2}([0, \pi], \mathbb{R}),
$$

determined by the one-dimensional wave operator

$$
u_{t t}-u_{x x}, \quad u(\cdot, 0)=u(\cdot, \pi)=0,
$$

we have $\lambda_{i}=i^{2} \quad \forall i$. Then (1.3) is satisfied provided $\alpha$ has a bounded continued fraction expansion [1,2]. Unfortunately, the author does not know generally such a nice criterion for $\alpha$ in (1.3). On the other hand, we have

Theorem 1.2. Assume

$$
\sum_{\lambda_{i}>0} \frac{1}{\sqrt{\lambda_{i}}}<\infty
$$

Then the Lebesque measure of the set of all positive $\alpha$ not satisfying (1.2) is zero.

This theorem says nothing for the case

$$
A u=-u_{x x}, \quad u(0)=u(\pi)=0, \quad u \in C^{2}([0, \pi], \mathbb{R}) .
$$

But taking

$$
\begin{aligned}
A u=u_{x x x x}, \quad u(0) & =u(\pi)=0, \quad u_{x x}(0)=u_{x x}(\pi)=0, \\
u & \in C^{4}([0, \pi], \mathbb{R}),
\end{aligned}
$$

we have $\lambda_{i}=i^{4} \quad \forall i$, and Theorem 1.2 holds. The operator $u_{t t}+u_{x x x x}$ appears in the equation of a beam.

In the second part of the paper, we assume both $\operatorname{ker} A \neq\{0\}$, i.e., $\lambda_{k}=0$ for some $k$, and $\operatorname{dim} \operatorname{ker} A<\infty$. We shall study this case as above in the framework of the bifurcation theory.

\section{NONRESONANT CASE}

First, we shall specify the equation (1.1). Hence $A: X \rightarrow Y$ is a bounded, linear operator, $X$ is a Banach space, and $Y$ is a Hilbert one with an inner product $\langle\cdot, \cdot\rangle$. Moreover, we assume that eigenvalues $\left\{u_{j}\right\}_{1}^{\infty}$ of $A$ corresponding to $\left\{\lambda_{i}\right\}_{1}^{\infty}$ satisfy

(a) $u_{j} \in X \quad \forall j$

(b) $\left\{u_{j}\right\}_{1}^{\infty}$ is an orthonormal basis of $Y$.

Furthermore, $f: Y \times S^{T} \rightarrow Y$ is continuous and globally Lipschitz in $y$ with the constant $M$. Here $S^{T}=\mathbb{R} /[0, T]$ is the circle.

In this section we study the case when

$$
\lambda_{i} \neq 0 \quad \forall i \text {. }
$$


A weak $T$-periodic solution of $(1.1)$ is some $u \in L^{2}\left(S^{T}, Y\right)$ satisfying

$$
\begin{gathered}
\int_{0}^{T}\left\langle u(t), v_{t t}(t)+A v(t)\right\rangle d t=\varepsilon \int_{0}^{T}\langle f(u(t), t), v(t)\rangle d t \\
\forall v \in C^{2}\left(S^{T}, X\right) .
\end{gathered}
$$

We mean the integrability in the sense of Bochner [3]. By (2.1) the Hilbert space $L^{2}\left(S^{T}, Y\right)$ has the orthogonal basis

$$
\begin{aligned}
&\left\{\sin m \frac{2 \pi t}{T} \cdot u_{j},\right.\left.\cos m \frac{2 \pi t}{T} \cdot u_{j}\right\} \subset C^{2}\left(S^{T}, X\right) \\
& m=0,1,2, \cdots, j=1,2, \cdots .
\end{aligned}
$$

Lemma 2.1. Assume (1.2). Then the equation

$$
\int_{0}^{T}\left\langle u(t), v_{t t}(t)+A v(t)\right\rangle d t=\int_{0}^{T}\langle h(t), v(t)\rangle d t \quad \forall v \in C^{2}\left(S^{T}, X\right)
$$

has a unique solution $u \in L^{2}\left(S^{T}, Y\right)$ for any $h \in L^{2}\left(S^{T}, Y\right)$. Moreover,

$$
\|u\|_{L^{2}\left(S^{T}, Y\right)} \leq c .\|h\|_{L^{2}\left(S^{T}, Y\right)}
$$

for a constant $c>0$.

Proof. By using both the Fourier expansion of $h$ in the basis (2.2) and the condition (1.2), we immediately obtain the proof. We point out that trigonometric polynomials of (2.2) are dense in $C^{2}\left(S^{T}, X\right)$.

The proof of Theorem 1.1 follows obviously by Lemma 2.1 together with the Banach fixed point theorem.

Proof of Theorem 1.2. If (1.2) is false for some $\alpha \in(K, K+1), K>0$, then for any $d>0$ small there exist $m, \lambda_{i}>0$ such that

$$
\left|\alpha^{2}-\frac{m^{2}}{\lambda_{i}}\right| \leq \frac{d}{\lambda_{i}}
$$

This implies

$$
\left|\alpha-\frac{m}{\sqrt{\lambda_{i}}}\right| \leq \frac{d}{K \lambda_{i}} .
$$

Since $\alpha \in(K, K+1)$, we have $\frac{m^{2}}{\lambda_{i}}<(K+1)^{2}+1$ for $d$ small. Thus

$$
m \leq \sqrt{\left((K+1)^{2}+1\right) \lambda_{i}}
$$

Denote by $\mathscr{M}$ the set of all $\alpha \in(K, K+1)$ for which (1.2) does not hold. Then the Lebesque measure $\mu(\mathscr{K})$ of $\mathscr{M}$ satisfies

$$
\mu(\mathscr{M}) \leq \sum_{\lambda_{i}>0} \frac{2 d}{\lambda_{i} K} \cdot \sqrt{\left((K+1)^{2}+1\right) \lambda_{i}}=\frac{2 d}{K} \sqrt{\left((K+1)^{2}+1\right)} \sum_{\lambda_{i}>0} \frac{1}{\sqrt{\lambda_{i}}} .
$$

Since $d$ is arbitrarily small, $\mu(\mathscr{M})=0$. The proof is finished. 


\section{Resonant CASE}

Assume the assumptions for $A, f$ from $\S 2$ hold, without (n), since we suppose

$$
\exists \lambda_{k}=0 \text { and } \operatorname{dim} \operatorname{ker} A<\infty .
$$

By (W) any weak $T$-periodic solution of (1.1) satisfies

$$
\int_{0}^{T}\left\langle f(u(t), t), u_{p}\right\rangle d t=0 \quad \forall u_{p} \in \operatorname{ker} A .
$$

Then Lemma 2.1 holds as well for this case but only for $h$ such that

$$
\int_{0}^{T}\left\langle h(t), u_{p}\right\rangle d t=0 \quad \forall u_{p} \in \operatorname{ker} A .
$$

Let $P: Y \rightarrow \operatorname{ker} A$ be the orthogonal projection. Then (1.1) has the form

$$
\begin{aligned}
w_{t t}+A w & =\varepsilon Q f\left(w+u_{p}, \cdot\right), \\
0 & =\tilde{P} f\left(w+u_{p}, \cdot\right)
\end{aligned}
$$

where

$$
Q=I-\tilde{P}, \quad \tilde{P} h=\frac{1}{T} \int_{0}^{T} P h(t) d t, \quad w \in \operatorname{im} Q, \quad u_{p} \in \operatorname{ker} A .
$$

Note that these projections are defined for the following reason:

$$
\tilde{P} h=0 \Longleftrightarrow\left\{\begin{array}{l}
\int_{0}^{T}\left\langle h(t), u_{p}\right\rangle d t=0 \\
\forall u_{p} \in \operatorname{ker} A .
\end{array}\right.
$$

Now, we are able to solve the first equation of (3.2) for $\varepsilon$ small as in $\S 2$. By inserting this solution $w\left(\varepsilon, u_{p}\right)$ into the second equation of $(3.2)$ we arrive at the bifurcation equation

$$
Q\left(\varepsilon, u_{p}\right)=\tilde{P} f\left(w\left(\varepsilon, u_{p}\right)+u_{p}, \cdot\right) .
$$

Note

$$
\begin{aligned}
& Q\left(0, u_{p}\right)=\frac{1}{T} \int_{0}^{T} P f\left(u_{p}, t\right) d t, \\
& Q(0, \cdot): \operatorname{ker} A \rightarrow \operatorname{ker} A .
\end{aligned}
$$

Summing up we obtain

Theorem 3.1. Let (1.2) be satisfied. Assume $Q(0, \cdot)$ is $C^{1}$-smooth and there exists $u_{p_{0}} \in \operatorname{ker} A$ such that

$$
\begin{aligned}
& Q\left(0, u_{p_{0}}\right)=0, \\
& D Q\left(0, u_{p_{0}}\right) \text { is regular } .
\end{aligned}
$$

Then (1.1) has a weak $T$-periodic solution for any $\varepsilon$ small.

Proof. We have to solve $Q\left(\varepsilon, u_{p}\right)=0$. By the assumptions of Theorem 3.1 this equation has a solution near $u_{p_{0}}$ for any $\varepsilon$ small. Indeed, the Leray-Schauder 
degree $\operatorname{deg}\left(Q(0, \cdot), B_{\delta}, 0\right)$ of $Q(0, \cdot)$ at $u_{p_{0}}$ is well defined for $\delta>0$ small, since $Q(0, \cdot)$ is a local isomorphism. Here

$$
B_{\delta}=\left\{z \in \operatorname{ker} A \mid\left\langle z-u_{p_{0}}, z-u_{p_{0}}\right\rangle=\delta^{2}\right\} .
$$

Moreover, this degree is nonzero. The proof is finished.

Example. Consider

$$
\begin{aligned}
& u_{t t}-u_{x x}-n^{2} u=\varepsilon f(u, t), \\
& u(t+T, \cdot)=u(t, \cdot) \quad \forall t, \\
& u(t, 0)=u(t, \pi)=0 \quad \forall t,
\end{aligned}
$$

where $f: \mathbb{R} \times S^{T} \rightarrow \mathbb{R}$ is $C^{1}$-smooth and globally Lipschitz in $u$ and $n \in \mathscr{N}$.

Now, we have for this case

$$
\begin{aligned}
& A u=-u_{x x}-n^{2} u, u(0)=u(\pi)=0, \\
& \operatorname{ker} A=\{\sin n x\}, \quad \lambda_{i}=i^{2}-n^{2}, \\
& X=\left\{u \in C^{2}([0, \pi], \mathbb{R}) \mid u(0)=u(\pi)=0\right\}, \quad Y=L^{2}([0, \pi], \mathbb{R}),
\end{aligned}
$$

and the condition (1.2) reads as follows:

$$
\left|\alpha^{2}-\frac{m^{2}}{i^{2}-n^{2}}\right| \geq \frac{c}{i^{2}-n^{2}} \quad \forall m, i \in \mathcal{N}, i>n,
$$

for a constant $c>0$. Note that (3.4) is equivalent to

$$
\left|\frac{i^{2}-n^{2}}{m^{2}}-\frac{1}{\alpha^{2}}\right| \geq \frac{c}{\alpha^{2} m^{2}} \quad \forall m, i \in \mathscr{N}, i>n,
$$

for a constant $c>0$.

Theorem 3.2. The equation (3.3) has a weak solution, provided (3.5) holds and there is $z \in \mathbb{R}$ such that

$$
\begin{aligned}
& \int_{0}^{T} \int_{0}^{\pi} f(z \cdot \sin n x, t) \sin n x d x d t=0 \\
& \int_{0}^{T} \int_{0}^{\pi} \frac{\partial f}{\partial u}(z \cdot \sin n x, t) \sin ^{2} n x d x d t \neq 0 .
\end{aligned}
$$

Proof. We see that for this case

$$
\begin{aligned}
& P u=\frac{2}{\pi} \int_{0}^{\pi} u(x) \sin n x d x \cdot \sin n x, \\
& Q\left(0, u_{p}\right)=\frac{2}{T \pi} \int_{0}^{T} \int_{0}^{\pi} f(z \cdot \sin n x, t) \sin n x d x d t \cdot \sin n x, \\
& u_{p}=z \cdot \sin n x .
\end{aligned}
$$

Now applying Theorem 3.1, the proof is finished.

In the last part of this section, we find a criterion similar to [1, 2] for $\alpha$ which ensures that $\alpha$ will satisfy (3.4)-(3.5), since these conditions are vague. We see (3.5) is equivalent to

$$
\inf _{i, m \in \mathcal{N}, i>n}\left|i^{2}-n^{2}-\omega^{2} m^{2}\right|>0
$$


where $\omega=1 / \alpha$. We put $q=i-n>0$ and obtain from (3.6)

$$
\inf _{q, m \in \mathscr{N}}\left|q^{2}+2 q n-\omega^{2} m^{2}\right|>0 .
$$

We compute

$$
\begin{aligned}
\left|q^{2}+2 q n-\omega^{2} m^{2}\right| & =m^{2}\left|\left(\frac{q}{m}\right)^{2}+2 \frac{q}{m} n-\omega^{2}\right| \\
& =m^{2}\left|\frac{q}{m}-\gamma_{1}\right| \cdot\left|\frac{q}{m}-\gamma_{2}\right|,
\end{aligned}
$$

where

$$
\gamma_{1,2}=-n \pm \sqrt{n^{2}+\omega^{2}}
$$

are the roots of $z^{2}+2 z n-\omega^{2}$. We see $\gamma_{1}>0, \gamma_{2}<0$. Hence

$$
\left|q^{2}+2 q n-\omega^{2} m^{2}\right| \geq m^{2}\left|\gamma_{2}\right| \cdot\left|\gamma_{1}-\frac{q}{m}\right| \text {. }
$$

By using the result of $[1,2]$ we know that, if $\gamma_{1}$ has a bounded continued fraction expansion, then there exists a constant $c>0$ satisfying

$$
\left|\gamma_{1}-\frac{q}{m}\right| \geq \frac{c}{m^{2}} \quad \forall q, m \in \mathcal{N} .
$$

This implies

$$
\inf _{q, m \in \mathscr{N}}\left|q^{2}+2 q n-\omega^{2} m^{2}\right|>0 .
$$

Note

$$
\gamma_{1}=\sqrt{n^{2}+\omega^{2}}-n, \quad \omega=1 / \alpha,
$$

i.e.,

Summarizing we obtain

$$
\alpha=\frac{1}{\sqrt{\gamma_{1}^{2}+2 \gamma_{1} n}} .
$$

Proposition 3.3. Assume

$$
\alpha=\frac{1}{\sqrt{\gamma^{2}+2 \gamma n}}
$$

for an irrational number $\gamma>0$ possessing a bounded continued fraction expansion. Then the number $\alpha$ satisfies the condition (3.4).

\section{REFERENCES}

1. K. Ben-Naoum and J. Mawhin, The periodic-Dirichlet problem for some semilinear wave equations, J. Differential Equations 96 (1992), 340-354.

2. P. J. McKenna, On solutions of a nonlinear wave equation when the ratio of the period to the length of the interval is irrational, Proc. Amer. Math. Soc. 93 (1985), 59-64.

3. H. Gajewski, K. Gröger, and K. Zacharias, Nichtlineare Operatorgleichungen und Operatordifferentialgleichungen, Akademie-Verlag, Berlin, 1974.

Mathematical Institute, Slovak Academy of Sciences, Śtefánikova 49, 81473 BratiSLAVA, SLOVAKIA

E-mail address: matefeckesavba.savba.sk 